\title{
Challenges and opportunities for development of an AIDS vaccine
}

\begin{abstract}
Gary J. Nabel
Vaccine Research Center, National Institutes of Allergy and Infectious Diseases, National Institutes of Health, 40 Convent Drive, Bethesda, Maryland 20892-3005, USA (e-mail: gnabel@nih.gov)

Among the devastating consequences of AIDS has been its epidemic spread in the developing world. The disease has caused unprecedented suffering, debilitation, loss of life and disruption of family, social and economic stability. Because of the considerable expense and logistical difficulty in providing antiviral drugs to populations infected with the human immunodeficiency virus throughout the world, the biomedical community is looking towards vaccines to help solve this compelling problem.
\end{abstract}

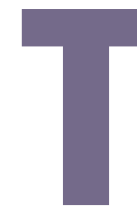
he search for an AIDS vaccine began more than 15 years ago with great optimism and high expectations. With the identification of the human immunodeficiency virus (HIV) as the cause of AIDS, it seemed that a vaccine would follow closely behind. But despite a large concerted effort, the problem has proven more difficult than anticipated, and progress has not matched the initial hopes. Here I review the principal scientific obstacles confronting the development of an effective HIV vaccine, and I consider potential strategies to overcome these obstacles.

\section{The challenge}

It is instructive to consider the circumstances that have contributed to past successes in vaccine development. The smallpox vaccine is among the most successful interventions in the history of medicine. Why, 200 years ago, without the benefit of modern biotechnology, did the smallpox vaccine succeed so readily while an AIDS vaccine remains elusive? The answer lies in an experiment of nature that provided, to an astute observer, a clear direction for smallpox vaccine development. In this classic story of scientific discovery, Edward Jenner noticed that milkmaids who had previously contracted cowpox were resistant to smallpox infection. This observation was the critical event leading to the finding that the cowpox virus cross-reacted immunologically with the smallpox virus and could therefore be used to protect against smallpox.

Jenner's milkmaids represented a protected population that provided the key information needed to develop the vaccine. Unfortunately, there are no significant large populations with well-defined resistance to HIV infection, and thus no immune parameters have been identified that correlate with protection. A cohort of exposed seronegative sex workers has been identified in Nairobi ${ }^{1}$, and it was hoped that this group could provide information about immune resistance to the virus. Unfortunately, protection is not always long lasting, the mechanism of their resistance has not been clearly identified, and thus they have not yielded definitive markers to guide vaccine development.

Development of an HIV vaccine is possible without the benefit of a correlate of immunity or a surrogate marker of protection in advance, but the road to its discovery will be considerably more arduous. The lack of immune correlates remains one of the more compelling challenges for the development of an AIDS vaccine (Table 1). The identification of immunogens that induce broad and long-lasting immunity has been another critical hurdle. And the genetic diversity of the virus poses still another difficulty: HIV displays an unusual degree of diversity that confounds efforts to create a vaccine that is universally effective against the various clades and viral strains ${ }^{2}$. In contrast, the strain variation of other viruses, such as poliovirus, for which vaccines have succeeded, is relatively limited. For example, a vaccine from three strains of poliovirus has been used successfully worldwide ${ }^{3,4}$.

Progress in HIV vaccine research is also subject to the limitations of animal models. Lentiviral infection of non-human primates provides an unusually good animal model for viral vaccine development that has provided important insights into HIV immunopathogenesis. But despite many similarities in the symptoms and pathology, there remain distinctions that could affect vaccine efficacy. For example, the simian immunodeficiency viruses (SIV) differ significantly from their human counterparts. With regard to genomic organization, the SIV viral accessory protein Vpx is not found in HIV-1, the Vpu gene product of HIV-1 is not found in SIV (reviewed in ref. 5), and the functions of $\mathrm{Vpr}$ also differ between the two viruses. Although some HIV strains have been shown to cause disease in chimpanzees, these primates seem to be largely resistant to the CCR5-tropic primary isolates responsible for infection of most humans, ${ }^{6}$, suggesting species differences in the host response to virus. The characteristics of molecular clones and laboratory-adapted viruses also differ from naturally infectious virus. Thus, despite the attractive features of the model, it is evident that human clinical studies will be needed for the development of effective vaccines. Such trials require the production of clinical-grade vaccines and requisite safety and toxicity studies, which pose additional challenges. As trials progress, the most important task is to choose the promising candidates for phase III clinical trials that test the efficacy of a vaccine based on limited information.

\section{The immune response to HIV vaccines}

The immune system responds to infectious agents through the elaboration of a humoral response in which B lymphocytes produce secreted antibodies that interact with a variety of infectious agents, including bacteria, viruses, fungi and parasites. In addition, cell-mediated immune responses induce helper $\mathrm{T}$ lymphocytes that stimulate antibody responses and cytotoxic Tlymphocytes (CTLs), which recognize processed antigens and lyse infected cells. Both of 
these immune mechanisms can be manipulated in vaccine development and each has its advantages and disadvantages (Table 2).

\section{Cell-mediated immunity}

CTLs are also known as killer T cells because they recognize, bind and kill cells that display foreign antigens. Their role in protective immunity against viral infections has been well documented ${ }^{7,8}$. An immunogen that elicits a CTL response enables the recognition and elimination of infected cells, and a CTL response is highly desirable in an AIDS vaccine because CTLs can eliminate or reduce virus production by killing viral producer cells. The importance of CTL responses in limiting viral infection has been supported by human studies and by studies of nonhuman primates ${ }^{1,9,10}$. Potent CTL responses and resistance to HIV was seen in the exposed seronegative Nairobi sex workers described above ${ }^{1}$. And in experiments with chronic SIV-infected rhesus macaques, a marked increase in viral load was seen when $\mathrm{CD} 8^{+} \mathrm{T}$ cells were depleted experimentally in these animals ${ }^{9,10}$.

Despite the importance of CTLs in containing viral replication, uncertainties remain as to whether cell-mediated immunity will be necessary and sufficient for a highly effective vaccine. Whether CTLs can be maintained in an active state for long periods of time or can expand rapidly enough to respond when an acute viral insult occurs is uncertain. Furthermore, vaccines that depend strictly on a CTL response may be countered by viral adaptations that allow the virus to evade detection by T cells. HIV, for example, has evolved mechanisms to disrupt the major histocompatibility complex (MHC) proteins, the antigen-presenting proteins that $\mathrm{T}$ cells rely on to recognize foreign antigens on the cell surface. One of these mechanisms involves the HIV gene product Nef, which downregulates expression of CD4 and class I MHC, cell-surface proteins that are essential to CTL recognition of viral antigens on viral producer cells ${ }^{11-13}$.

\section{Humoral immunity}

Because CTL responses alone are unlikely to provide complete protection, it will be important for an HIV vaccine to elicit neutralizing antibodies to the virus. Such antibodies are dependent on memory $\mathrm{B}$ cells, a long-lived cell population that can divide and differentiate into the antibody-producing plasma cell upon re-exposure to antigen, thus conferring long-term protection. Another advantage of antibodies is that they have the potential to inactivate virus before it has a chance to infect the cells of the host. Antibodies may also mobilize the inflammatory system, including the complement system, neutrophils and monocytes. Thus, even when an antibody does not directly neutralize the virus, there is potential by other antibody effector functions for amplification through the inflammatory system.

Although the antibody response should be a critical factor in antiviral immunity, one of the main hurdles for a highly effective HIV vaccine has been the development of immunogens that elicit broadly neutralizing antibodies. It has been possible to generate antibodies against the envelope protein of HIV, but such antibodies have limited efficacy. This problem arises, in part, because they neutralize laboratory-adapted strains but are not effective against primary isolates and are often strain-specific ${ }^{14}$. Although several broadly neutralizing monoclonal antibodies that neutralize the infectivity of different strains have been identified, it has not been possible to elicit this response with well-defined immunogens (reviewed in refs 15-18). The extensive genetic diversity among different strains and clades of HIV has created considerable difficulty in this regard. In addition, there have been suggestions that some antibodies may enhance infectivity ${ }^{19-21}$, and there are indeed precedents for this complication in vaccine development. In efforts to develop vaccines against the respiratory syncytial virus, some early vaccine candidates were found to exacerbate infection ${ }^{22,23}$. It is clear that antibody responses must be assessed carefully.

The ability of antibodies to confer protection at mucosal sites is also of great importance to the development of an effective AIDS vaccine. Several studies have investigated the ability of antibodies to protect against infection from intravenous challenge ${ }^{14}$ and through mucosal surfaces, with encouraging results. Using chimaeric SIV/HIV viruses (SHIVs) in non-human primates, Mascola and colleagues infused neutralizing antibodies into rhesus macaques to protect against vaginally transmitted infection ${ }^{24}$, while Baba and coworkers tested the effects of such antibodies in an oral mucosal exposure after birth ${ }^{25}$. Passive transfer of antibodies conferred protection against disease in both studies. Although relatively high concentrations of antibody $\left(\geqslant 100 \mu \mathrm{g} \mathrm{ml}^{-1}\right)$ were used - levels much higher than would ordinarily be achieved by vaccination - these studies showed that an appropriate serum antibody response might reduce infection at mucosal surfaces. It is unlikely that vaccination could achieve such a robust antibody response, although vaccines could also generate cellular immunity that might reduce the requisite neutralizing antibody concentration to protect against infection. It is also hoped that synergy between antibodies directed against different neutralizing determinants might reduce the concentration required for effective neutralization.

\section{Replication-defective viral vaccines}

The best example of protection against infection by a lentivirus (the retroviral genus of the HIV species) involves the use of a live-attenuated virus. Desrosiers and colleagues showed that monkeys infected with SIV, attenuated by the deletion of the viral gene nef, were not infected upon subsequent challenges with wild-type or nef-deleted $\mathrm{SIV}^{26}$. However, despite the initial excitement generated by these promising results, additional observations indicated potential hazards of this approach. It was eventually found that significant pathology occurred in both infant and adult macaques after exposure to attenuated SIV s7-29. In addition, there have been several reports of patients infected with HIV containing naturally occurring mutations in nef or the regulatory region of the long terminal repeat. Initially these patients exhibited less aggressive disease progression, but over time they were found to have reduced CD4 counts and increased viral loads $s^{30-32}$. Although live-attenuated viruses may prove ultimately to be effective and safe, many concerns remain to be addressed before these viruses will be acceptable for use in human clinical trials.

\section{Multi-component immunity for an effective vaccine}

In the absence of known immune correlates of protection, it would be most prudent to develop a vaccine that stimulates multiple components of the immune system ${ }^{33}$. The logical conclusion from the extensive literature on immune protection in lentiviral infection is that a combination of long-lived memory $\mathrm{T}$ cells, both $\mathrm{CD} 8^{+}$CTLs and $\mathrm{CD}^{+}$memory helper $\mathrm{T}$ cells, will probably be needed for a highly effective AIDS vaccine. At the same time, a strategy to induce broadly neutralizing antibodies will be required for highly effective, long-lasting immunity. It is now also evident that the $\beta$-chemokine receptors are necessary for HIV infection (reviewed in refs 34, 35). In fact, one of the most compelling examples of natural immunity to HIV is found in individuals with mutations in this receptor ${ }^{36,37}$. Thus, immunogens that induce antibodies which disrupt $\beta$-chemokinereceptor binding may prove to be useful.

In summary, although the immune correlates of protection remain unknown, there is evidence that cell-mediated immunity controls viral replication. At the same time, evidence from other successful vaccine approaches has indicated that long-term B-cell memory, through the antibody response, is crucial in immune protection. The challenge is to develop a vaccine that can elicit a broadly reactive $\mathrm{T}$-cell response that is long lasting, and to identify antigens that will elicit the 'correct' (broadly neutralizing) antibody response.

\section{Recombinant vector vaccines and adjuvants}

A variety of vaccine strategies that use inactive viruses or individual viral components have been investigated ${ }^{38}$. The original and still most common immunogens used in vaccines are live-attenuated or inactivated viruses. Viruses attenuated from a pathogenic agent have proven 


Table 1 Summary of the main hurdles in development of an AIDS vaccine
1. Identification of immunogens that induce broad and long-lasting CTL immunity
2. Definition of structures and immunization strategies that elicit broadly neutralizing
antibodies
3. Definition of immune correlates of protection in human or animal models
4. Strategies to address HIV clade and strain diversity
5. Expansion of human clinical trials:
Clinical-grade vaccine production
Diversity and duration of immune response
Prioritization and analysis of candidates

safe and effective in many widely used vaccines, including the Sabin poliovirus and chickenpox vaccine. In these cases, a non-pathogenic attenuated virus elicits an immune response that cross-reacts immunologically with the virulent virus. More recently, replicationdefective viral vectors unrelated to the pathogen have been used to deliver selected viral immunogens that might induce protective immunity. Another new approach has been the use of genetically engineered plasmid DNA to direct the synthesis of an immunogen within the host cells (reviewed in refs 39, 40). Combinations of these approaches, such as DNA priming and viral vector boosting, seem to be especially promising in animal models of vaccine development.

\section{Nonviral vectors}

DNA vaccines contain a gene encoding a viral immunogen under the regulation of a eukaryotic enhancer/promoter and polyadenylation signals that confer appropriate expression of the viral immunogen. The various elements of DNA vaccines can be readily manipulated to optimize the level and duration of expression and the potency of the immunogen. When injected into muscle, cells surrounding the injection site internalize the plasmid and transport the DNA to the nucleus where transcription, translation and appropriate posttranslational modification occur. Compared to recombinant protein vaccines produced in bacteria or yeast, the proteins expressed from a DNA vaccine are more likely to assume a native conformation, and their localized production facilitates uptake by antigen-presenting cells. Thus, antibodies generated against these immunogens are more likely to recognize and provide protection against native nondenatured proteins of the pathogen. In addition, antigen synthesis within cells will lead to more effective class I MHC processing and presentation that should stimulate CTL responses.

DNA vaccines could alleviate some potential disadvantages of live-attenuated virus vaccines such as the possibility of pathogenic infection and side effects of chronic immune stimulation. The feasibility of genetic immunization has been shown in several experimental model systems ${ }^{41-43}$. Furthermore, in rodents it has proven effective in inducing immunity to a variety of infectious diseases including influenza virus ${ }^{44}$, malaria ${ }^{45-48}$, tuberculosis ${ }^{49}$, Ebola virus ${ }^{50}$, rabies $^{51}$, lymphocytic choriomeningitis virus $s^{52,53}$ and herpes simplex virus ${ }^{54}$. Studies of non-human primates and humans have indicated that this approach is particularly effective in generating CTL responses ${ }^{55}$. A recent study showed that rhesus monkeys receiving a DNA vaccine augmented with a recombinant interleukin-2 plasmid developed potent CTL responses and did not develop clinical disease after a pathogenic SHIV challenge ${ }^{56}$. This was particularly significant not only for the success with a DNA vaccine strategy but also because vaccination prevented the appearance of disease symptoms.

Despite promising results in rodent models, DNA vaccinations have proven less effective in primates. It is likely that this technical issue can be overcome with time and increased experience, and several approaches have been taken to improve these vaccines. For example, more potent gene-expression strategies that use stronger enhancers or gene amplification have been explored. Another approach has been to modify the transcriptional and translational efficiency of foreign DNA by using codon choices preferred in the host species ${ }^{57,58}$. The use of human codons in some gene-based vectors, particularly from viruses and other infectious agents, increases the level of production significantly, most likely by modifying RNA regulatory structures that prevent export from the nucleus to the cytoplasm, thus preventing effective full-length translation and transcription. By overcoming these blocks and optimizing expression, more antigen is available to present to the immune system, which may prove helpful in eliciting more effective immunity to HIV.

\section{Viral vectors}

From efforts in vaccine development and gene therapy, new viral vectors have been advanced in recent years that may prove useful for AIDS vaccines. These include replication-defective forms of poxvirus vectors, including canarypox, fowlpox and modified vaccinia Ankara ${ }^{59-61}$. Highly immunogenic viruses, such as adenovirus, may also prove useful, particularly when strains are identified that are not reactive with antibodies commonly found in humans. Other vectors have progressed through pre-clinical development, including Sindbis ${ }^{62,63}$ and Venezuelan equine encephalitis virus al phaviruses ${ }^{64,65}$. Replication-defective vectors from these viruses synthesize high levels of recombinant protein and can target delivery to dendritic cells. In addition, vectors have been identified that may allow persistent transgene expression to stimulate continuous T-cell activation. Advances in lentiviral vector development have shown that they can be modified to address a number of safety concerns $s^{66-68}$. Adeno-associated virus has also proven effective in achieving long-term gene expression and is the subject of research for AIDS vaccines ${ }^{69,70}$.

\section{Adjuvants}

Key to the development of any successful vaccine is the use of adjuvants that augment immune responses to specific antigens. Adjuvants have traditionally been defined as substances used in combination with a specific antigen to elicit a more potent immunity than when the antigen is used by itself. A variety of adjuvants have been tested in animal models and human studies, and the subject is the topic of intense interest on which several insightful texts and reviews have been written ${ }^{71-75}$. With respect to HIV vaccines, several adjuvants have been tested in phase I clinical trials. These include polymers such as oligolysine, lipopeptides and polylactide co-polymers ${ }^{76}$. Traditional adjuvants such as aluminium phosphate or aluminium hydroxide, which are precipitated with the antigen, QS-21, MF59, monophosphoryl lipid A, mineral oil, mannose mono-oleate or incomplete Freund's adjuvant, purified protein derivative, keyhole limpet haemocyanin and bupivicaine (for DNA vaccines) have been analysed, with varying degrees of efficacy (summarized in ref. 76). In addition, a number of innovative technologies have been adapted, including the use of cytokine proteins or cytokine DNA expression vectors, immunostimulatory DNA sequences and the formulation of new complexes designed to create microparticles that can facilitate uptake of antigen-presenting cells.

The mechanisms by which these adjuvants work are not fully understood, but suggestions include: (1) preferential stimulation of specific T-cell subsets; (2) targeting of antigen to antigen-presenting cells; (3) direction of antigen into the MHC class I or class II pathways; (4) antigen deposition with slow release; and (5) stabilization of epitopes. A number of factors can affect the efficacy of adjuvants. These variables are related to the mode of administration, formulation of the adjuvant, species-specific responses to the immunostimulatory effects of the adjuvant, and the immune status of the host. So far, there is no clear preferential adjuvant for use in HIV vaccines. This has already been the topic of considerable investigation in phase I studies by the Vaccine Trials Network supported by the US National Institutes of Health and promises to be a continued area of important research for the development of highly effective vaccines.

\section{Choice of immunogens}

HIV encodes more than 12 gene products, any of which might serve as targets for immune recognition. The synthesis of these viral proteins is regulated by viral transactivators and includes proteins derived from messenger RNAs synthesized from highly spliced viral 
RNA, made early after the course of infection, and those derived from unspliced viral RNA, produced late in the viral life cycle. Several of these viral proteins contribute to the structure of virus and are synthesized in high quantities, such as the matrix and capsid proteins. Others give rise to regulatory proteins that modulate viral gene expression and are synthesized in lower quantities. Because CTLs are more likely than antibodies to be effective against internal viral proteins, attention has focused on the use of Gag proteins as immunogens for the CTL response. For Env, which is found on the surface of the virus, even though CTL responses are likely to be beneficial, the accessibility of this protein on intact virions would make it an attractive target for neutralizing antibodies. These two products of late, unspliced viral RNAs are widely considered to be important constituents of a highly effective HIV vaccine.

Among the highly spliced, early viral RNA products, Nef is expressed at high levels, and its importance in viral replication has been demonstrated ${ }^{77}$, although it is uncertain whether it will be an effective target for vaccine. Because it is expressed early in the virus life cycle, immune responses to this protein may serve to limit the burst size of virus from individual cells and could contribute to protective immunity. Finally, the Tat and Rev regulatory proteins have been the focus of study because of their potent regulatory activity. The Rev protein is found primarily in the nucleus but is not expressed at high levels; because it is not highly immunogenic it is not considered an attractive vaccine candidate. In contrast, the Tat protein, although also found in the nucleus at low levels, has been described in extracellular tissues, and several investigators have suggested that it may exert biological effects relevant to the pathogenesis of HIV disease (reviewed in ref. 78). Several laboratories have explored the potential utility of Tat as a constituent of an AIDS vaccine. Although reports have indicated that modified forms of Tat proteins can induce immune responses that may reduce viral replication in models using non-human primates ${ }^{79,80}$, it is not yet clear whether these models are relevant to natural infection in humans ${ }^{81}$. The protective effects induced by immunization with modified Tat proteins have not been observed with gene-based Tat delivery, and these findings have raised questions about the ultimate efficacy of Tat as an immunogen. Whether this difference is due to alternative immune responses to Tat by protein versus genetic immunization, to specific features of the primate models of infection, or to other undefined variables remains unclear and will require further investigation.

\section{Immune evasion by HIV}

The challenges involved in developing an HIV vaccine go beyond issues of optimizing expression. Indeed, simply generating antibodies is no challenge. Rather, the difficulty lies in the identification of immunogens that stimulate the production of broadly neutralizing antibodies. There are a number of reasons why this has been so difficult, and they involve the many adaptations that HIV has evolved to thwart the immune system.

Because almost every infected individual generates an antibody response, HIV is a virus that has been selected in the presence of antibodies. The viral envelope is the part of the virus most accessible to the immune system. Thus, it has evolved under this selective pressure to evade immune detection (reviewed in ref. 82). This is accomplished in several ways, such as glycosylation of envelope proteins, and masking of critical parts of proteins including CD4 and co-receptor binding sites. In addition, the envelope itself seems to be conformationally active ${ }^{82}$. Thus, some structures that need to be recognized at the time the virus engages its receptor are not exposed to the immune system at a time when they could be accessible to B cells or antibody. It is also possible that the envelope has developed decoy mechanisms. For example, the protein includes epitopes that may attract an immune response that diverts recognition from highly conserved regions of the envelope critical for receptor binding and entry. HIV can also escape detection by the cellular immune response through multiple mechanisms, for example, by Nef-mediated reduction in class I MHC expression or through complex gene regulation that permits latent infection of cells.

\section{Structure-function relationships in immunogen design}

Clearly, gaining the advantage over HIV requires a more thorough understanding of viral envelope structure. X-ray crystallographic studies ${ }^{83-86}$ have provided valuable information on the structures of the envelope proteins, gp120 and gp41. The crystal structure of gp120, for example, reveals numerous mechanisms of immune evasion, including conformational change, steric occlusion, islands of variation and a carbohydrate cloak ${ }^{82}$. Analysis of gp120 in complex with $\mathrm{CD} 4$, the primary virus receptor, for example, reveals that the CD4 binding site is recessed and contains several cavities. Many residues critical for antibodies directed against this region are not accessible in the CD4-bound conformation, although the rim of the conserved 'Phe 43 cavity' may be accessible for neutralization ${ }^{82}$. It is likely that the native envelope protein will need to be altered as an immunogen to effectively present epitopes that can elicit neutralizing antibody responses. Other potential molecular targets include structures that may be exposed only transiently during fusion and entry. Alternatively, it may be possible to elicit one antibody that induces a conformational change in the envelope spike that would expose otherwise cryptic sites (for example, normally masked chemokine-receptor binding surfaces on gp120) to attack by a second antibody.

The viral envelope has intrinsic immunogenicity, and when it is injected into its recipient, it tends to induce a certain stereotypical immune response. Different components of the virus generate different responses. For example, when mice are injected with DNA encoding gp160, a CTL response is readily generated. However, if antibody production is examined in the same mice, the titre is found to be very low, whereas another viral gene, nef, induces high titre antibodies and low CTL activity. It is important to understand the genetic and structural bases for divergent immune responses, for example, by the analysis of the immunogenicity of diverse mutant envelope proteins. The ultimate goal is to combine immunogenicity information with an analysis of the physical structure of these mutant proteins. Although this information may differ depending on the vector and/or adjuvant, such understanding of structure and its relation to immunogenicity may help to identify promising vaccine candidates.

Another structural approach to vaccine design has derived from analysis of the mechanism of viral fusion and the HIV gp41 region

\begin{tabular}{|c|c|c|}
\hline & Advantages & Disadvantages \\
\hline CTL-eliciting vaccines & $\begin{array}{l}\text { Recognition of virally } \\
\text { infected cells } \\
\text { Multiple linear epitopes } \\
\text { Elimination of virus } \\
\text { production } \\
\text { Possible effect on latent } \\
\text { reservoir }\end{array}$ & $\begin{array}{l}\text { Requirement for active, } \\
\text { long-term memory cells } \\
\text { Inability to recognize virus in } \\
\text { absence of MHC } \\
\text { Down-modulation of MHC } \\
\text { by virus }\end{array}$ \\
\hline Antibody-eliciting vaccines & $\begin{array}{l}\text { Neutralization of virus } \\
\text { Ability to prevent new } \\
\text { infection of cells } \\
\text { Activation of inflammatory } \\
\text { response (complement } \\
\text { system, neutrophils } \\
\text { and monocytes }\end{array}$ & $\begin{array}{l}\text { Inability to generate broadly } \\
\text { neutralizing antibodies } \\
\text { Evolution of resistant strains } \\
\text { Antibodies may enhance } \\
\text { infectivity to exacerbate } \\
\text { infection }\end{array}$ \\
\hline $\begin{array}{l}\text { Replication-defective } \\
\text { viral vaccines }\end{array}$ & $\begin{array}{l}\text { Persistent antigen } \\
\text { expression } \\
\text { Induction of cell-mediated } \\
\text { and humoral immunity } \\
\text { Interference }\end{array}$ & $\begin{array}{l}\text { Safety and inadvertent infection } \\
\text { Enhanced replication during } \\
\text { immune suppression } \\
\text { Consequences of persistent } \\
\text { immune stimulation } \\
\text { Increased rates of integration }\end{array}$ \\
\hline
\end{tabular}


that contains structures relevant to this process. Fusion requires two triple helical coiled-coil regions that fold like a hairpin to generate a six helical bundle ${ }^{85,86}$. The helical coiled-coil structure facilitates insertion of the fusion peptide into its target cell. This structure is not unique to HIV, as it is found in a variety of viruses, including influenza virus, murine leukaemia viruses, SIV and respiratory syncitial virus, and within eukaryotic cells in the soluble NSF attachment receptor (SNARE) involved in vesicle fusion (summarized in ref. 87).

Matthews, Hunter and Bolognesi ${ }^{88}$ and Kim and co-workers ${ }^{89}$ have shown that a peptide that interferes with the helical coiled-coil structure of HIV gp41 disrupts viral fusion and decreases viral load in human clinical studies. Efforts have been made to focus on this structure as a target for neutralization by antibodies, but, unfortunately, antibodies against this structure do not seem to neutralize the virus. It is likely that once the six-helical coiled-coil structure has formed, it is not accessible to antibodies. Thus, another approach has been to target intermediate structures that form before the hairpin has been generated. Nunberg and co-workers used this approach in experiments where mice were immunized with complexes of Env-expressing cells admixed with $\mathrm{CD}^{+} / \mathrm{CCR}^{+}$target cells in an effort to form fusion intermediates ${ }^{90}$. Although promising in the reported study, this method has been difficult to apply to primate models and will prove challenging for largescale vaccine production. Thus, many laboratories continue to address the critical problem of immunization with fusion intermediates using alternative molecular approaches. It is not yet known whether such envelope proteins can be identified, but through a combination of DNA technology and structural information, it will be possible to rapidly create and survey a variety of immunogens that are both informative and useful in vaccine development.

\section{Current and future directions for AIDS vaccine research}

The challenges faced in the development of an AIDS vaccine are similar to those that remain for a number of medically important infectious diseases, including malaria and tuberculosis. In all cases, safe, effective, broad-spectrum vaccines are needed that generate long-term immune memory and protection at the sites of infection, particularly at mucosal sites where the virus gains entry into the body. Because the vaccines are needed in parts of the world where the most modern medical care is not necessarily available, it will also be necessary to have simple, transportable vectors that can be administered easily.

HIV vaccines have been evaluated so far in over 70 phase I (dose-escalation safety and toxicity), five phase II (expanded safety and dose optimization) and two phase III (efficacy) clinical trials. These studies have evaluated safety and immunogenicity of preventive vaccines in more than 3,500 subjects. Among them, several envelope proteins, which have been made in insect, yeast or mammalian cells with recombinant DNA technology and which encode gp120 or gp160, have been administered with different adjuvants. Peptides derived from the envelope V3 loop or Gag have been delivered as conjugates to oligolysine backbones, as lipopeptide conjugates, with alum, or as fusion proteins with the self-assembling yeast protein Ty as a particle. Alternative sites of delivery have been analysed, including intramuscular, oral and rectal routes. In addition, gene-based vectors, such as vaccinia, canarypox and salmonella, as well as nucleic acid-based vaccines, have been tested. These studies are reviewed in detail elsewhere ${ }^{76,91,92}$. No significant safety concerns have arisen. Although neutralizing antibody responses have been detected, their activity on primary CCR5-tropic isolates has been minimal and has largely been strain-specific ${ }^{93,94} . \mathrm{CD}^{+} \mathrm{CTL}$ responses have also been found that are durable, with some crossclade reactivity ${ }^{95}$, although the consistency of such responses is not yet optimal. Data on the efficacy of any of these vaccines in human populations are not yet available. Most researchers believe that a highly promising ideal vaccine candidate is not at hand, and that it will be necessary to evaluate additional candidates that generate strong, broad and long-lasting CTL and neutralizing antibody responses.
There are numerous scientific opportunities to enhance vaccine development. Methods must be developed to enhance the immunogenicity of specific HIV peptides and to enhance antibody responses to regions of HIV proteins that are naturally protected from immune detection. These regions are generally the most conserved, so antibodies recognizing them would be most likely to be more broadly effective across the various clades and strains of HIV. Improved understanding of adjuvants is also needed.

Another important tool for vaccine development is the rapidly evolving field of genomics. An understanding of the human genome as well as the viral genome will help to identify genetic factors that determine immunological responders and confer immunological resistance. Analysis of the gene-expression profiles and linkage studies with polymorphic human genetic markers during vaccine trials and in association with HIV infection will facilitate identification of genes that confer resistance to infection, and may help to determine which vaccines are best suited to specific populations.

Considerable work remains to be done in the area of translation from animal studies to clinical trials. New vectors and more efficient methods for the production of vectors are needed. The most important aspect of translation is to understand the response of the immune system to vaccines. The original antibody and CTL assays have been invaluable in this regard, but they are relatively time consuming and cumbersome. Newer and more efficient technologies are being developed that provide detailed information about T-cell-receptor specificities and cytokine profiles ${ }^{96}$. These will be essential for identification and implementation of new vaccine candidates.

Human clinical studies remain the critical link between laboratory research and an effective vaccine. Vital to this effort is an informative network of clinical trials, both in the developed and developing world. Also crucial is the successful collaboration of the public and private sector in establishing the knowledge base, production expertise and vaccine distribution network that will someday be required for a successful vaccine. The search for an HIV vaccine has been slow and at times frustrating, but the resolve of the biomedical research community to address this problem has grown. Although the solution is not yet at hand, progress is tangible and encouraging results now develop on a regular basis. This renewed commitment and these advances in the science of AIDS vaccine development will make it possible to meet this unprecedented challenge.

Rowland-Jones, S. L. et al. Cytotoxic T cell response to multiple conserved HIV epitopes in HIVresistant prostitutes in Nairobi. J. Clin. Invest. 102, 1758-1765 (1998).

2. Saag, M. S. et al. Extensive variation of human immunodeficiency virus type-1 in vivo. Nature 334, 440-444 (1988).

3. Melnick, J. L. in Fields Virology (eds Fields, B. N., Knipe, D. M. \& Howley, P. M.) 655-712 (LippincottRaven, Philadelphia, 1996)

4. Chanock, R. M. Reminiscences of Albert Sabin and his successful strategy for the development of the live oral poliovirus vaccine. Proc. Assoc. Am. Phys. 108, 117-126 (1996).

5. Luciw, P. A. in Fields Virology (eds Fields, B. N., Knipe, D. M. \& Howley, P. M.) 1881-1952 (Lippincott-Raven, Philadelphia, 1996).

6. Novembre, F. J. et al. Development of AIDS in a chimpanzee infected with human immunodeficiency virus type 1. J. Virol. 71, 4086-4091 (1997).

7. Zinkernagel, R. \& Doherty, P. Major transplantation antigens, viruses, and specificity of surveillance T cells. Contemp. Top. Immunobiol. 7, 179-220 (1977).

8. Zinkernagel, R. \& Doherty, P. MHC-restricted cytotoxic T cells: studies on the biological role of polymorphic major transplantation antigens determining T-cell restriction-specificity, function, and responsiveness. Adv. Immunol. 27, 51-177 (2000).

9. Jin, X. et al. Dramatic rise in plasma viremia after $\mathrm{CD} 8^{+} \mathrm{T}$ cell depletion in simian immunodeficiency virus-infected macaques. J. Exp. Med. 189, 991-998 (1999).

10. Schmitz, J. E. et al. Control of viremia in simian immunodeficiency virus infection by CD8 lymphocytes. Science 283, 857-860 (1999).

11. Collins, K. L., Chen, B. K., Kalams, S. A., Walker, B. D. \& Baltimore, D. HIV-1 Nef protein protects infected primary cells against killing by cytotoxic T lymphocytes. Nature 391, 397-401 (1998).

12. Schwartz, O., Marechal, V., Le Gall, S., Lemonnier, F. \& Heard, J. M. Endocytosis of major histocompatibility complex class I molecules is induced by the HIV-1 Nef protein. Nature Med. 2, 338-342 (1996).

13. Cullen, B. R. HIV-1 auxiliary proteins: making connections in a dying cell. Cell 93, 685-692 (1998).

14. Mascola, J. R. et al. Protection of macaques against pathogenic simian/human immunodeficiency virus 89.6PD by passive transfer of neutralizing antibodies. J. Virol. 73, 4009-4018 (1999).

15. Parren, P. W., Burton, D. R. \& Sattentau, Q. J. HIV-1 antibody—debris or virion? Nature Med. 3, 366-367 (1997).

16. Sattentau, Q. J. Neutralization of HIV-1 by antibody. Curr. Opin. Immunol. 8, 540-545 (1996). 
17. Burton, D. R. A vaccine for HIV type 1: the antibody perspective. Proc. Natl Acad. Sci. USA 94, 10018-10023 (1997)

18. Burton, D. R. \& Montefiori, D. C. The antibody response in HIV-1 infection. AIDS 11(Suppl. A), S87-S98 (1997)

19. Morens, D. M. Antibody-dependent enhancement of infection and the pathogenesis of viral disease. Clin. Infect. Dis. 19, 500-512 (1994).

20. Burke, D. S. Human HIV vaccine trials: does antibody-dependent enhancement pose a genuine risk? Perspect. Biol. Med. 35, 511-530 (1992).

21. Mascola, J. R. et al. Summary report: workshop on the potential risks of antibody-dependent enhancement in human HIV vaccine trials. AIDS Res. Hum. Retroviruses 9, 1175-1184 (1993).

22. Johnson, P. R. \& Collins, P. L. Sequence comparison of the phosphoprotein mRNAs of antigenic subgroups A and B of human respiratory syncytial virus identifies a highly divergent domain in the predicted protein. J. Gen. Virol. 71, 481-485 (1990).

23. Kim, H. W. et al. Respiratory syncytial virus disease in infants despite prior administration of antigenic inactivated vaccine. Am. J. Epidemiol. 89, 422-434 (1969).

24. Mascola, J. R. et al. Protection of macaques against vaginal transmission of a pathogenic HIV-1/SIV chimeric virus by passive infusion of neutralizing antibodies. Nature Med. 6, 207-210 (2000).

25. Baba, T. W. et al. Human neutralizing monoclonal antibodies of the IgG1 subtype protect against mucosal simian-human immunodeficiency virus infection. Nature Med. 6, 200-206 (2000)

26. Daniel, M. D., Kirchhoff, F., Czajak, S. C., Sehgal, P. K. \& Desrosiers, R. C. Protective effects of a live attenuated SIV vaccine with a deletion in the nef gene. Science 258, 1938-1941 (1992).

27. Baba, T. W. et al. Pathogenicity of live, attenuated SIV after mucosal infection of neonatal macaques. Science 267, 1820-1825 (1995).

28. Baba, T. W. et al. Live attenuated, multiply deleted simian immunodeficiency virus causes AIDS in infant and adult macaques. Nature Med. 5, 194-203 (1999).

29. Wyand, M. S. et al. Protection by live, attenuated simian immunodeficiency virus against heterologous challenge. J. Virol. 73, 8356-8363 (1999).

30. Deacon, N. J. et al. Genomic structure of an attenuated quasi species of HIV-1 from a blood transfusion donor and recipients. Science 270, 988-991 (1995).

31. Greenough, T. C., Sullivan, J. L. \& Desrosiers, R. C. Declining CD4 T-cell counts in a person infected with nef-deleted HIV-1. N. Engl. J. Med. 340, 236-237 (1999).

32. Learmont, J. C. et al. Immunologic and virologic status after 14 to 18 years of infection with an attenuated strain of HIV-1. N. Engl. J. Med. 340, 1715-1722 (1999).

33. Dittmer, U., Brooks, D. M. \& Hasenkrug, K. J. Requirement for multiple lymphocyte subsets in protection by a live attenuated vaccine against retroviral infection. Nature Med. 5, 189-193 (1999).

34. Gallo, R. C., Garzino-Demo, A. \& DeVico, A. L. HIV infection and pathogenesis: what about chemokines? J. Clin. Immunol. 19, 293-299 (1999).

35. Garzino-Demo, A., DeVico, A. L., Cocchi, F. \& Gallo, R. C. Beta-chemokines and protection from HIV type 1 disease. AIDS Res. Hum. Retroviruses 14(Suppl. 2), S177-S184 (1998),

36. Dean, M. et al. Genetic restriction of HIV-1 infection and progression to AIDS by a deletion allele of the CCR5 structural gene. Science 273, 1856-1862 (1996).

37. Samson, M. et al. Resistance to HIV-1 infection in Caucasian individuals bearing mutant alleles of the CCR-5 chemokine receptor gene. Nature 382, 722-725 (1996).

38. Letvin, N. L. Progress in the development of an HIV-1 vaccine. Science 280, 1875-1880 (1998)

39. Donnelly, J. J., Ulmer, J. B., Shiver, J. W. \& Liu, M. A. DNA vaccines. Annu. Rev. Immunol. 15, 617-648 (1997).

40. Tighe, H., Corr, M., Roman, M. \& Raz, E. Gene vaccination: plasmid DNA is more than just a blueprint. Immunol. Today 19, 89-97 (1998).

41. Tang, D. C., DeVit, M. \& Johnston, S. A. Genetic immunization is a simple method for eliciting an immune response. Nature 356, 152-154 (1992).

42. Liu, M. A. et al. Vaccination of mice and nonhuman primates using HIV-gene-containing DNA. Antibiot. Chemother. 48, 100-104 (1996).

43. Wang, B. et al. DNA inoculation induces protective in vivo immune responses against cellular challenge with HIV-1 antigen-expressing cells. AIDS Res. Hum. Retroviruses 10(Suppl. 2), S35-S41 (1994).

44. Ulmer, J. B. et al. Heterologous protection against influenza by injection of DNA encoding a viral protein. Science 259, 1745-1749 (1993).

45. Becker, S. I. et al. Protection of mice against Plasmodium yoelii sporozoite challenge with P. yoelii merozoite surface protein 1 DNA vaccines. Infect. Immun. 66, 3457-3461 (1998).

46. Doolan, D. L. et al. Circumventing genetic restriction of protection against malaria with multigene DNA immunization: $\mathrm{CD}^{+}$cell-, interferon gamma-, and nitric oxide-dependent immunity. J. Exp. Med. 183, 1739-1746 (1996).

47. Gardner, M. J. et al. DNA vaccines against malaria: immunogenicity and protection in a rodent model. J. Pharm. Sci. 85, 1294-1300 (1996).

48. Sedegah, M., Hedstrom, R., Hobart, P. \& Hoffman, S. L. Protection against malaria by immunization with plasmid DNA encoding circumsporozoite protein. Proc. Natl Acad. Sci. USA 91, 9866-9870 (1994).

49. Tascon, R. C. et al. Vaccination against tuberculosis by injection. Nature Med. 2, 888-892 (1996).

50. Xu, L. et al. Immunization for Ebola virus infection. Nature Med. 4, 37-42 (1998).

51. Lodmell, D. L. et al. DNA immunization protects nonhuman primates against rabies virus. Nature Med. 4, 949-952 (1998).

52. Martins, L. P., Lau, L. L., Asano, M. S. \& Ahmed, R. DNA vaccination against persistent viral infection. J. Virol. 69, 2574-2582 (1995).

53. Yokoyama, M., Zhang, J. \& Whitton, J. L. DNA immunization confers protection against lethal lymphocytic choriomeningitis virus infection. J. Virol. 69, 2684-2688 (1995).

54. Manickan, E., Yu, Z., Rouse, R. J., Wire, W. S. \& Rouse, B. T. Induction of protective immunity against herpes simplex virus with DNA encoding the immediate early protein ICP 27. Viral Immunol. 8, 53-61 (1995).

55. Wang, R. et al. Induction of antigen-specific cytotoxic T lymphocytes in humans by a malaria DNA vaccine. Science $282,476-480$ (1998).

56. Barouch, D. H. et al. Control of viremia and prevention of clinical AIDS in rhesus monkeys by cytokine-augmented DNA vaccination. Science 290, 486-492 (2000).

57. Schwartz, S. et al. Mutational inactivation of an inhibitory sequence in human immunodeficiency virus type 1 results in Rev-independent gag expression. J. Virol. 66, 7176-7182 (1992).
58. Andre, S. et al. Increased immune response elicited by DNA vaccination with a synthetic gp 120 sequence with optimized codon usage. J. Virol. 72, 1497-1503 (1998).

59. Tartaglia, J. et al. Canarypox virus-based vaccines: prime-boost strategies to induce cell-mediated and humoral immunity against HIV. AIDS Res. Hum. Retroviruses 14(Suppl. 3), S291-S298 (1998).

60. Moss, B. et al. Host range restricted, non-replicating vaccinia virus vectors as vaccine candidates. $A d v$. Exp. Med. Biol. 397, 7-13 (1996).

61. Tartaglia, J., Pincus, S. \& Paoletti, E. Poxvirus-based vectors as vaccine candidates. Crit. Rev. Immunol. 10, 13-30 (1990)

62. Wahlfors, J. J., Zullo, S. A., Loimas, S., Nelson, D. M. \& Morgan, R. A. Evaluation of recombinant alphaviruses as vectors in gene therapy. Gene Ther. 7, 472-480 (2000).

63. Huang, H. V. Sindbis virus vectors for expression in animal cells. Curr. Opin. Biotechnol. 7, 531-535 (1996).

64. Davis, N. L. et al. Vaccination of macaques against pathogenic simian immunodeficiency virus with Venezuelan equine encephalitis virus replicon particles. J. Virol. 74, 371-378 (2000).

65. Caley, I. J. et al. Venezuelan equine encephalitis virus vectors expressing HIV-1 proteins: vector design strategies for improved vaccine efficacy. Vaccine 17, 3124-3135 (1999).

66. Naldini, L. et al. In vivo gene delivery and stable transduction of nondividing cells by a lentiviral vector. Science 272, 263-267 (1996).

67.Zufferey, R. et al. Self-inactivating lentivirus vector for safe and efficient in vivo gene delivery. J. Virol. 72, 9873-9880 (1998)

68. Trono, D. Lentiviral vectors: turning a deadly foe into a therapeutic agent. Gene Ther. 7, 20-23 (2000).

69. Monahan, P. E. \& Samulski, R. J. AAV vectors: is clinical success on the horizon? Gene Ther. 7 , 24-30 (2000).

70. Liu, X. L., Clark, K. R. \& Johnson, P. R. Production of recombinant adeno-associated virus vectors using a packaging cell line and a hybrid recombinant adenovirus. Gene Ther. 6, 293-299 (1999).

71. Spriggs, D. R. \& Koff, W. C. Topics in Vaccine Adjuvant Research (CRC, Boca Raton, FL, 1991).

72. Powell, M. F. \& Newman, M. J. Vaccine Design: The Subunit and Adjuvant Approach (Plenum, New York, NY, 1995)

73. Cooper, P. D. in Strategies in Vaccine Design (ed. Ada, G. L.) 125-158 (Landes, Austin, TX, 1994).

74. Cox, J. C. \& Coulter, A. R. Adjuvants-a classification and review of their modes of action. Vaccine 15, 248-256 (1997)

75. O'Hagan, D. T. Vaccine Adjuvants: Preparation Methods and Research Protocols (Humana Press, Totowa, NJ, 2000).

76. Graham, B. in HIV Molecular Immunology Database (eds Korber, B. T. et al.) (Los Alamos National Laboratory: Theoretical Biology and Biophysics, Los Alamos, NM, 2000).

77. Kestler, H. W. III et al. Importance of the nef gene for maintenance of high virus loads and for development of AIDS. Cell 65, 651-662 (1991).

78. Emerman, M. \& Malim, M. H. HIV-1 regulatory/accessory genes: keys to unraveling viral and host cell biology. Science 280, 1880-1884 (1998).

79. Cafaro, A. et al. Control of SHIV-89.6P-infection of cynomolgus monkeys by HIV-1 Tat protein vaccine. Nature Med. 5, 643-650 (1999).

80. Pauza, C. D. et al. Vaccination with tat toxoid attenuates disease in simian/HIV-challenged macaques. Proc. Natl Acad. Sci. USA 97, 3515-3519 (2000).

81. Travis, J. A controversial shot in the arm. Science News 156, 300-303 (1999).

82. Wyatt, L. \& Sodroski, J. The HIV-1 envelope glycoproteins: fusogens, antigens, and immunogens. Science 280, 1884-1888 (1998).

83. Kwong, P. D. et al. Structure of an HIV gp 120 envelope glycoprotein in complex with the CD4 receptor and a neutralizing human antibody. Nature 393, 648-659 (1998).

84. Kwong, P. D. Structures of HIV-1 gp 120 envelope glycoproteins from laboratory-adapted and primary isolates. Structure 8, 1329-1339 (2000).

85. Chan, D. C., Fass, D., Berger, J. M. \& Kim, P. S. Core structure of gp41 from the HIV envelope glycoprotein. Cell 89, 263-273 (1997).

86. Weissenhorn, W., Dessen, A., Harrison, S. C., Skehel, J. J. \& Wiley, D. C. Atomic structure of the ectodomain from HIV-1 gp41. Nature 387, 426-430 (1997).

87. Weissenhorn, W., Carfi, A., Lee, K. H., Skehel, J. J. \& Wiley, D. C. Crystal structure of the Ebola virus membrane fusion subunit, GP2, from the envelope glycoprotein ectodomain. Mol. Cell 2, 605-616 (1998).

88. Kilby, J. M. et al. Potent suppression of HIV-1 replication in humans by T-20, a peptide inhibitor of gp41-mediated virus entry. Nature Med. 4, 1302-1307 (1998).

89. Eckert, D. M., Malashkevich, V. N., Hong, L. H., Carr, P. A. \& Kim, P. S. Inhibiting HIV-1 entry: discovery of D-peptide inhibitors that target the gp41 coiled-coil pocket. Cell 99, 103-115 (1999).

90. LaCasse, R. A. et al. Fusion-competent vaccines: broad neutralization of primary isolates of HIV. Science 283, 357-362 (1999).

91. Graham, B. S. \& Karzon, D. T. in Textbook of AIDS Medicine (eds Merigan, T. C. Jr, Bartlett, J. G. \& Bolognesi, D.) 689-724 (Lippincott, Williams, and Wilkins, Baltimore, MD, 1998).

92. Dolin, R. Human studies in the development of human immunodeficiency virus vaccines. J. Infect. Dis. 172, 1175-1183 (1995).

93. Wrin, T., Loh, T. P., Vennari, J. C., Schuitemaker, H. \& Nunberg, J. H. Adaptation to persistent growth in the $\mathrm{H} 9$ cell line renders a primary isolate of human immunodeficiency virus type 1 sensitive to neutralization by vaccine sera. J. Virol. 69, 39-48 (1995).

94. Mascola, J. R. et al. Immunization with envelope subunit vaccine products elicits neutralizing antibodies against laboratory-adapted but not primary isolates of human immunodeficiency virus type 1. J. Infect. Dis. 173, 340-348 (1996).

95. Ferrari, G. et al. Clade B-based HIV-1 vaccines elicit cross-clade cytotoxic T lymphocyte reactivities in uninfected volunteers. Proc. Natl Acad. Sci. USA 94, 1396-1401 (1997).

96. Pitcher, C. J. et al. HIV-1-specific CD4 ${ }^{+} \mathrm{T}$ cells are detectable in most individuals with active HIV-1 infection, but decline with prolonged viral suppression. Nature Med. 5, 518-525 (1999).

\section{Acknowledgements}

I thank B. Graham, P. Kwong, J. Mascola and R. Wyatt for helpful discussions and comments and T. Suhana, A. Tislerics and N. Barrett for assistance with preparation of the manuscript. 